\title{
Comment on "Prognostic Role of Claudin-1 Immunohistochemistry in Malignant Solid Tumors: A Meta-Analysis"
}

\author{
Bolin Wang, Yan Huang ${ }^{1}$ \\ Weifang Medical University, Weifang; 'Department of Oncology, Affiliated Hospital of Weifang Medical University, Weifang, China
}

\section{Dear Editor,}

With great interest, we read the article "Prognostic Role of Claudin-1 Immunohistochemistry in Malignant Solid Tumors: A Meta-Analysis". ${ }^{1}$ The authors stated that low claudin-1 immunohistochemistry expression is significantly correlated with worse survival in various malignant tumors. The results of the meta-analysis are encouraging. Nevertheless, there are still some shortcomings that need to be addressed.

Firstly, literature search is an important step in meta-analysis. If the collection is incomplete, it will lead to obvious selective bias. I want to know whether this is an independent collection in the process of literature collection and how the authors resolved the differences of opinion.

Secondly, the authors seem to have forgotten the key step: quality assessment. We doubt the quality of the attached literature. If the quality of the literature is poor, it will directly affect the value of the results.

Finally, overall survival and disease-free survival are two key endpoints. The authors point out that to avoid bias, articles with a follow-up date of 60 months were extracted. So we want to know if literature collection excluded articles that had follow-up period less than 60 months. Also, we want to know that the overall survival rate and disease-free survival rate refer to the 5 -year survival rate? If not, we recommend that it be shown in the text or table.

Nevertheless, we are grateful to the authors for their efforts in studying the association between claudin-1 immunohistochemistry and malignant solid tumors.

\section{ORCID}

Bolin Wang: https://orcid.org/0000-0001-8086-0681

Yan Huang: https://orcid.org/0000-0002-1599-9086

\section{Conflicts of Interest}

The authors declare that they have no potential conflicts of interest.

\section{REFERENCE}

1. Pyo JS, Kim NY, Cho WJ. Prognostic role of claudin-1 immunohistochemistry in malignant solid tumors: a meta-analysis. J Pathol Transl Med 2019; 53: 173-9. 